\title{
The measurement of the material parameters of viscoelastic fluids using a rotating sphere and a rheogoniometer
}

\author{
A. Acharya and P. Maaskant \\ With 3 figures
}

(Received November 8, 1977)

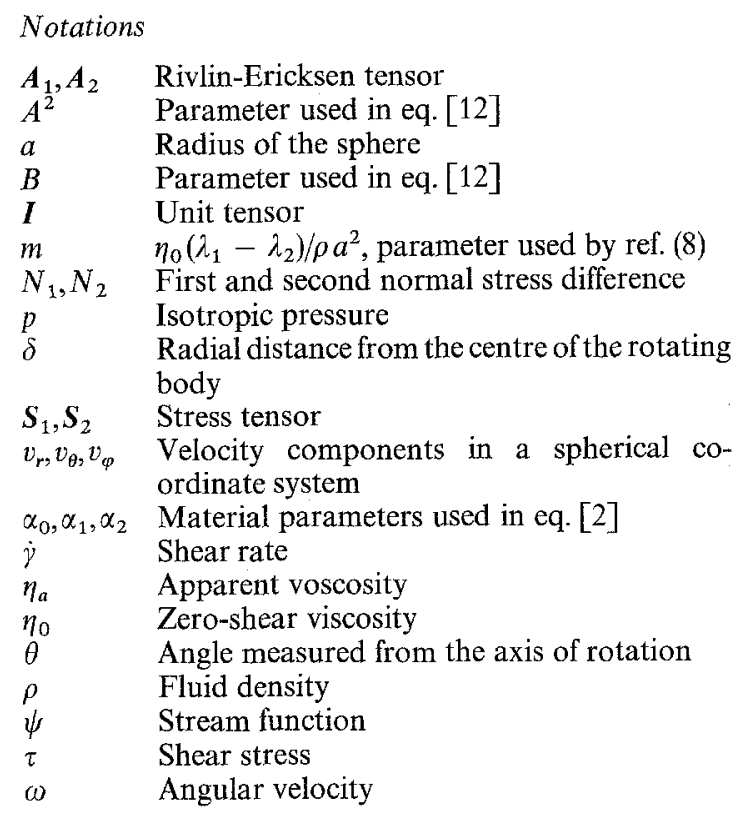

\section{Introduction}

A study of the literature on the measurement of rheological material parameters shows that viscometric flow arrangements (e.g. capillary flow, Couette flow, cone and plate flow etc.) have been widely used (11). However, in recent times it has become increasingly clear that viscometric flow arrangements alone are not sufficient for the complete evaluation of material parameters and to do this a combination of both viscometric and non-viscometric flow arrangements are useful.

Recently, there have been few attempts to determine the material parameters of viscoelastic fluid from non-viscometric flows in and around. Remove the period special geometries. These are given in a chronological order elsewhere (6). In our work, we attempted to obtain. material parameters using the flow of a viscoelastic fluid around a rotating sphere.

In both viscometric and non-viscometric flows, the material parameters can be obtained experimentally either by measuring an integral quantity (e.g. torque) or from the direct measurements of velocity distribution. For the later case, techniques involving stream line tracing with dye injection or particle tracer methods have been popular in the past. But recently there has been progress in the application of LaserDoppler anemometry for measuring flow fields without introducing any physical interference into the system such as hot wire probes or tracer particles.

\section{Theoretical}

\subsection{Constitutive Equation}

The constitutive equation of a second-order fluid can be written as (7),

$\tau=-p I+S_{1}+S_{2}+\cdots$

where,

$S_{1}=\alpha_{0} A_{1}$,

$S_{2}=\alpha_{1} A_{2}+\alpha_{2} A_{1}^{2}$.

In the above equation $\boldsymbol{A}_{1}$ and $\boldsymbol{A}_{2}$ are the first and second Rivlin-Ericksen tensors and $\alpha_{0}$ physically represents zero-shear viscosity $\left(\eta_{0}\right)$.

For a simple shear flow, the Rivlin-Ericksen tensors can be written in the following forms:

$S_{1}=\alpha_{0} \dot{\gamma}\left[N+N^{T}\right]$,

$S_{2}=\gamma^{2}\left[2 \alpha_{1} N^{\dagger} \cdot N+\alpha_{2}\left(N+N^{T}\right)^{2}\right]$,

and the three viscometric functions can be described in terms of material parameters. 
Shear stress:

$\tau(\dot{\gamma})=\alpha_{0} \dot{\gamma}$,

First Normal Stress Difference:

$N_{1}(\dot{\gamma})=-2 \alpha_{1} \dot{\gamma}^{2}$,

Second Normal Stress Difference:

$N_{2}(\dot{\gamma})=\left(2 \alpha_{1}+\alpha_{2}\right) \dot{\gamma}^{2}$.

\subsection{Flow Around a Rotating Sphere}

Consider a sphere of radius $a$ which rotates with an angular velocity $\omega$ about a vertical diameter in an infinite liquid media. A spherical polar co-ordinate $(r, \theta, \varphi)$ system is used in this work where $r$ is the distance measured from the centre of the sphere. For an axisymmetrical flow $\varphi$-component of velocity has a little importance in determining the velocity field. Number of attempts have been made in the past few years either to find out the potential use of a rotating sphere as a viscometer [see Mashelkar et al. (7), Walters and Savins (10), Hermes (4)] or to obtain the velocity distribution of a higher order fluid [see Giesekus $(2,3)]$.

In the present work we shall start with Giesekus' (2) solution for the stream function of a second-order Rivlin-Ericksen fluid.

In ref. (2) he solved the problem assuming that the effects of inertia can be neglected. $\mathrm{He}$ also calculated the secondary flow around a sphere as a special case and with the help of perturbation analysis he arrived at an expression for the stream function $\psi(r, \theta)$ as

$$
\begin{gathered}
\psi(r, \theta)=-\frac{a^{3} \omega^{2}}{8 \eta_{0}}\left[4\left(\alpha_{1}+\alpha_{2}\right)\left(1+\frac{2 a}{r}\right)\right] \\
\cdot\left(1-\frac{a}{r}\right)^{2} \sin ^{2} \theta \cos \theta .
\end{gathered}
$$

Taking the inertial effect into account from the perturbation flow analysis he found:

$$
\begin{gathered}
\psi(r, \theta)=\frac{a^{3} \omega^{2}}{8 \eta_{0}}\left[\rho a^{2}-4\left(\alpha_{1}+\alpha_{2}\right)\left(1+\frac{2 a}{r}\right)\right] \\
\cdot\left(1-\frac{a}{r}\right)^{2} \sin ^{2} \theta \cos \theta .
\end{gathered}
$$

The individual velocity components $v_{r}$ and $v_{\theta}$ can be written in terms of stream function as,

$v_{r}=-\frac{1}{r^{2} \sin \theta} \frac{\partial \psi}{\partial \theta}$,

$$
v_{\theta}=\frac{1}{r \sin \theta} \frac{\partial \psi}{\partial r} .
$$

Using eqs. [8] and [9], one obtains an expression for $v_{r}$ as

$$
\begin{aligned}
v_{r}= & \frac{a^{3} \omega^{2}}{8 r^{2} \eta_{0}}\left[\rho a^{2}-4\left(\alpha_{1}+\alpha_{2}\right)\left(1+\frac{2 a}{r}\right)\right] \\
& {\left[1-\frac{a}{r}\right]^{2}\left(1-3 \cos ^{2} \theta\right) . }
\end{aligned}
$$

At the equatorial plane $(\theta=\pi / 2)$, the radial velocity component $v_{r}$ becomes;

$v_{r}=\frac{A^{2}}{r^{2}}\left[1-B\left(1+\frac{2 a}{r}\right)\right]\left(1-\frac{a}{r}\right)^{2}$,

where

$A^{2}=\frac{\rho a^{5} \omega^{2}}{8 \eta_{0}}$ and $B=\frac{4\left(\alpha_{1}+\alpha_{2}\right)}{\rho a^{2}}$.

Here the dimensionless quantity, $B$ determines the shape of the velocity profile while $A^{2}$ determines the absolute value of the velocity. Secondary flow is a consequence of interaction between centrifugal forces (inertial effect) and elastic forces (normal stress effect) and the flow pattern varies with the value of $B$.

For $B \leq 1 / 3$, the centrifugal forces determine the nature of flow field and for $B \geq 1$, the elastic forces dominate where as for $1 / 3<B<1$, the elastic forces dominate near the surface of the sphere and further away from the sphere, the centrifugal forces dominate the flow situation. For $B>2$, the shape of the velocity profile becomes independent of the value of $B$. A complete picture of such flow situation is given in figure 1.

Thomas and Walters (8) have performed a similar analysis where they showed that the flow pattern of an Oldroyd fluid $\left(B^{\prime}\right)$ are strongly dependent on the liquid parameter $m=\eta_{0}\left(\lambda_{1}-\lambda_{2}\right) / \rho a^{2}$. When $m=0$ (Newtonian fluid) or less than $1 / 12$, the liquid is thrown out from the sphere at the equator and is directed outside at the poles, where as a reversal phenomena is observed for $1 / 12<m<1 / 4$.

\section{Experimental}

Experiments were carried out in a square $(60 \mathrm{~cm} \times 60 \mathrm{~cm})$ perspex tank containing $1 \%$ aqueous polyacryl amide (PAA, AP-30) solution.

The sphere (steel ball-bearing) suspended from a stainless steel shaft, was placed at the 
$B=0.3$

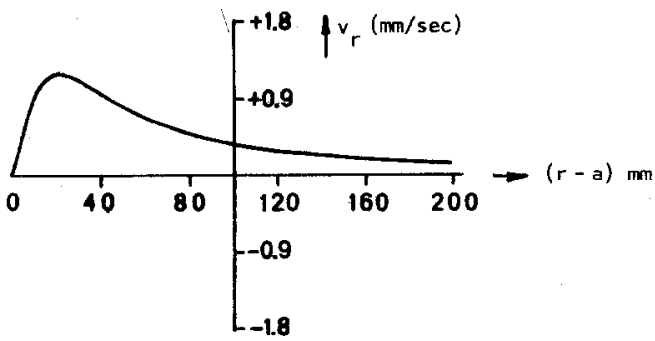

$B=0.6$

$B=2$
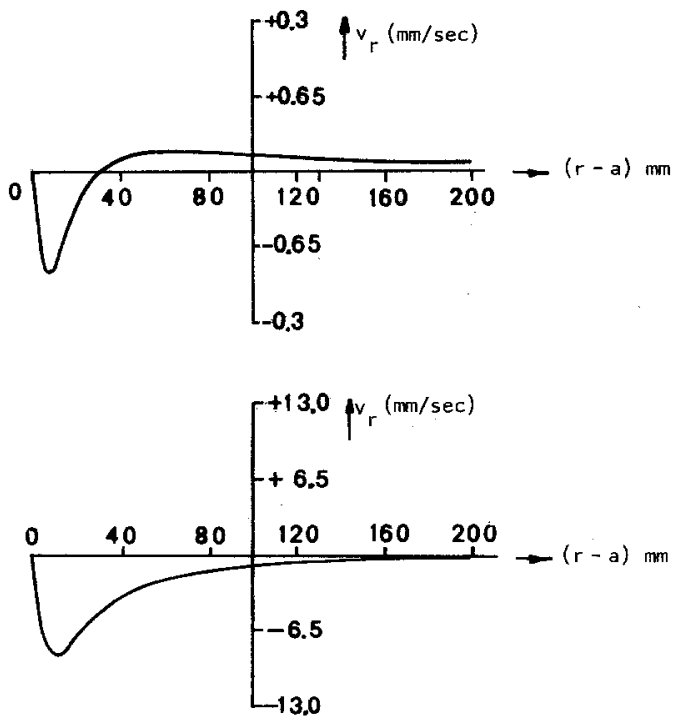

$B=10$

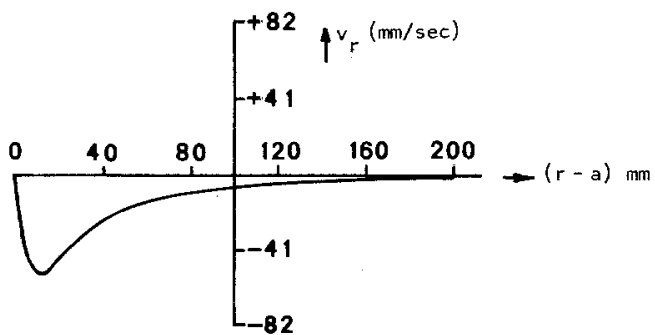

Fig. 1. A plot of $v_{r}$ vs. $(r-a)$ for different values of $B$ centre of the tank. The steel shaft was coupled with a step-variable electromotor (speed range 0.3-300 r.p.m.). Two different spheres (radii $15 \mathrm{~mm}$ and $25 \mathrm{~mm}$ ) were used for this particular experiment. The radii of the spheres were so chosen that the ratio of the maximum dimension of the container to the diameter of the sphere was of the order of $12: 1$ and consequently, the influence of the wall could be neglected [see Walters and Waters (9)].

Shear stress vs. shear rate and first normal stress difference (a measure of the total thrust experienced by the bottom plate of a cone and plate arrangement) vs. shear rate were obtained from the Weissenberg rheogoniometer (Model R-18). The actual velocity measurements were carried out by using a Laser-Doppler anemometer.

At the equatorial plane $(\theta=\pi / 2)$, apart from radial velocity there is also a tangential velocity. This tangential velocity, in general, is of much higher value. So in order to determine only the radial component of velocity, we had to eliminate the tangential velocity. This was done by choosing the measuring point exactly along the radial plane of the sphere so that the tangential velocity was directed along the optical axis and hence gives no Doppler contribution. This optimal position for radial component of velocity was found by trial and error method where the measuring point was shifted along the optical axis and the velocity was measured at every 
position for both clockwise and anti-clockwise rotation of the sphere. At the optimal position the radial velocity should remain the same for both the directions.

\section{Results and discussions}

\subsection{Viscometric measurements}

The experimental data obtained from the Weissenberg rheogoniometer were apparent viscosity vs. shear rates. The zero-shear viscosity $\left(\eta_{0}\right)$ was obtained by extrapolating the curve to zero-shear rate. This gave a value of $\eta_{0}$ around $20 \mathrm{~N} \cdot \mathrm{s} / \mathrm{m}^{2}$ which agrees reasonably well with the reported values of $\eta_{0}$ of about $22 \mathrm{~N} \cdot \mathrm{s} / \mathrm{m}^{2}$ for $1 \%$ PAA [see Caswell's fluidity plots (1), Mashelkar et al.'s semi-theoretical procedure with sphere and disc (5)].

\subsection{Non-viscometric measurements}

Radial velocity measurements have been performed around the rotating sphere at dif- ferent radial positions $(r-a)$ in a speed range 0.3 r.p.m. -15 r.p.m. using two different spheres having diameters of $3 \mathrm{~cm}$ and $5 \mathrm{~cm}$ respectively.

The experimental values of $v_{r}$ were then plotted against $(r-a)$ in figures 2 and 3 and the values of $A^{2}$ were calculated by using the estimated value of $\eta_{0}$. A least squares fit method was used to obtain the best fitting theoretical curve using Giesekus' expression (2) for $v_{r}$ (see eq. [12]) with the experimental data using $B$ as a fitting parameter. If the second-order Rivlin-Ericksen approximation is valid, then the best fitted value of $B$ for a known value of $A^{2}$ should give us the information about the material parameters $\left(\alpha_{1}+\alpha_{2}\right)$ for a second-order flow behavior. From figures 2 and 3 it is quite apparent that as one goes from 15 r.p.m. to 0.3 r.p.m., fitting of experimental points with that of the theoretical curve becomes much better and it is worth assuming that the second-order flow behavior have been obtained at a lower speed of the order of 0.3 r.p.m. So the values of $\left(\alpha_{1}+\alpha_{2}\right)$ obtained
(D) 1\% PAA, a $=15 \mathrm{~mm}$ $\omega=15$ r.p.m.

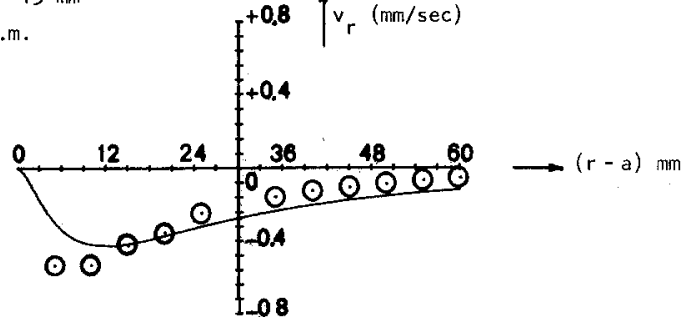

(E) 1\% PAA, $a=15 \mathrm{~mm}$ $\omega=3 r \cdot p \cdot m$.

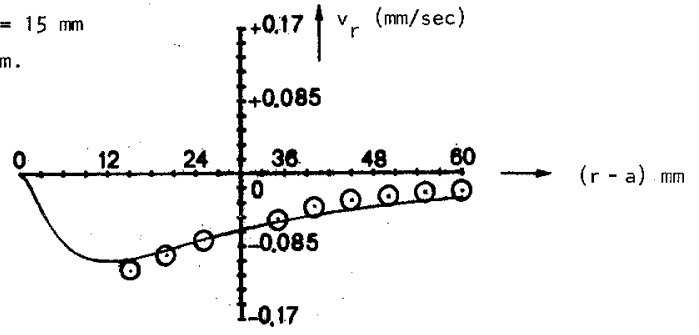

Fig. 2. A plot of $v_{r}$ vs. $(r-a)$ at different r.p.m. for a $3 \mathrm{~cm}$ sphere Theoretical curve $\odot \odot \odot$ Experimental curve 
(A) $1 \%$ PAA, a = $25 \mathrm{~mm}$
$\omega=15 \mathrm{r} . \mathrm{p} . \mathrm{m}$.

(B) $1 \%$ PAA, a $=25 \mathrm{~mm}$

$\omega=3$ r.p.m.

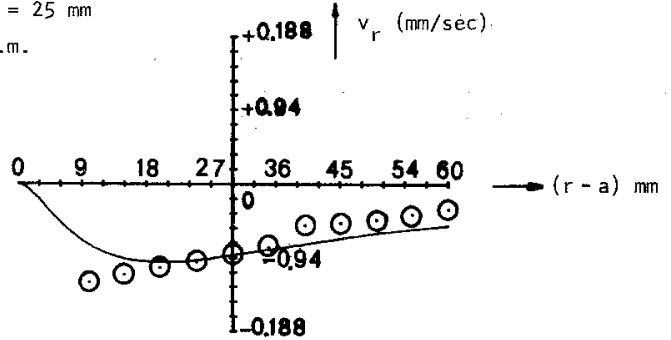

(c) $1 \%$ PAA, a $=25 \mathrm{~mm}$
$w=.3 \mathrm{r} \cdot \mathrm{p} . \mathrm{m}$.
Fig. 3. A plot of $v_{r}$ vs. $(r-a)$ at different r.p.m. for a $5 \mathrm{~cm}$ sphere Theoretical curve

$\odot \odot \odot$ Experimental curve for the sphere having a diameter of $3 \mathrm{~cm}$ was of the order of $300 \mathrm{~kg} / \mathrm{m}$. Whereas that of the sphere having a diameter of $5 \mathrm{~cm}$ was $400 \mathrm{~kg} / \mathrm{m}$. If the second-order fluid behavior had really been attained in each case, the value of $\left(\alpha_{1}+\alpha_{2}\right)$ should remain constant. The $25 \%$ difference between these two values could be involved with significant influence of fluid aging and finite shaft thickness $(8 \mathrm{~mm}$ in case of $3 \mathrm{~cm}$ sphere and $12 \mathrm{~mm}$ in case of $5 \mathrm{~cm}$ sphere).

In conclusion, it could be said that it seems to be possible to find the material parameters for a "second-order" fluid using a combination of viscometric and non-viscometric arrangements. In order to determine $\alpha_{2}$ separately, a different type of flow geometry is needed together with the present combination.

\section{Acknowledgements}

The authors wish to thank Department of Applied Physics, Technische Hogeschool Twente, The Netherlands, for providing all the facilities to carry out this research project and Dr. $R$. Jongschaap for his helpful comments on this work.

\section{Summary}

In this work, measurement of the flow field around a rotating sphere has been used to obtain the material parameters of a second-order Rivlin-Ericksen fluid. Experiments were carried out with a Laser-Doppler anemometer to obtain the velocity distribution and using Giesekus' analysis, the material parameters for the second-order fluid were obtained.

\section{Zusammenfassung}

In dieser Untersuchung wird die Ausmessung des Strömungsfeldes um eine rotierende Kugel dazu verwendet, um die Stoffparameter einer Rivlin-EricksenFlüssigkeit zweiter Ordnung zu erhalten. Die Experimente zur Bestimmung der Geschwindigkeitsverteilung werden mit einem Laser-Doppler-Anemometer durchgeführt, und zur Auswertung der Parameter der Flüssigkeit zweiter Ordnung wird eine Analyse von Giesekus benutzt. 


\section{References}

1) Caswell, B., unpublished work.

2) Giesekus, $H$., Proc. 4 th Int. Cong. Rheology Providence, 1963, 1, 249.

3) Giesekus, H., Rheol. Acta 4, 85 (1965). (1966).

4) Hermes, R. A., J. Appl. Polym. Sci. 10, 1793

5) Kelkar, J. V., R. A. Mashelkar, J. Ulbrecht, J. Appl. Polym. Sci. 17, 3069 (1973).

6) Maaskant, P., Baccalaureate Thesis, Technische Hogeschool Twente, The Netherlands (1977).

7) Mashelkar, R. A., D. D. Kale, J. Ulbrecht, J. Appl. Polym. Sci. 17, 973 (1973).

8) Thomas, R. G., K. Walters, Quart. J. Mech. and Appl. Math. 17, 39 (1964).

9) Walters, K., N. D. Waters, Brit. J. Appl. Phys. 15, 989 (1964).
10) Walters, K., J. G. Savins, Trans. Soc. Rheol. 9, 407 (1965).

11) Walters, K., Rheometry, John Wiley \& Sons, New York (1974).

Authors' addresses:

Dr. A. Acharya

Department of Chemical Engineering

Northwestern University

Evanston, Illinois 60201 (USA)

\section{P. Maaskant}

Department of Applied Physics

Technische Hogeschool Twente Enschede (The Netherlands) 